\title{
Frühkindliches Asthma: Doppellblindstudie mit inhalativem Budenosid
}

\author{
In einer randomisierten, plazebokontrollierten Doppelblindstudie \\ an 40 Kindern im Alter bis zu 30 Monaten mit schwerem Asthma \\ zeigte die inhalative Therapie mit Budenosid eine im Vergleich zu \\ Plazebo deutlich überlegene Wirksamkeit bei guter Verträglichkeit.
}

Die inhalative Kortikoidtherapie wird bei Erwachsenen und Kindern mit Asthma bronchiale als prophylaktische Maßnahme allgemein empfohlen. Allerdings führten die bisherigen Studien bei Kindern zu widersprüchlichen Ergebnissen: Nicht in allen Untersuchungen konnte eine Minderung der asthmatischen Symptome nachgewiesen werden.

Daher wurde jetzt von einer französisch-englischen Arbeitsgruppe der Effekt einer inhalativen Behandlung mit vernebeltem Budenosid erstmals in einer randomisierten, plazebokontrollierten Doppelblindstudie an einer - verglichen mit den bisherigen Studien - etwas größeren Stichprobe untersucht.

40 Kinder im Alter bis zu 30 Monaten mit schwerem Asthma bronchiale erhielten über 12 Wochen entweder zweimal täglich $1 \mathrm{mg}$ Budenosid oder Plazebo über einen Inhalator mit Vernebler. Danach folgte eine 12wöchige Follow-up-Phase. Die Basismedikation während der Studie bestand aus einer dreimal täglichen oralen Gabe von $100 \mu \mathrm{g} / \mathrm{kg}$ Salbutamol. Insgesamt kam es bei Kindern mit ständigem Asthma eher zu Exazerbationen als bei den Patienten mit intermittierendem Asthma. In der Budenosid-Gruppe hatten signifikant weniger Kinder mindestens eine Exazerbation (40\% vs. $83 \%$ in der Plazebo-Gruppe). Auch die Dauer der bei auftretenden Exazerbationen notwendigen oralen Kortikoidgabe war unter Budenosid signifikant geringer als unter Plazebo. Nach 12 Wochen waren in der Verumgruppe $55 \%$ der Kinder frei von Exazerbationen, unter Plazebo $8 \%$ $(\mathrm{p}<0,05)$. Die Häufigkeit des Giemens war unter Budenosid sowohl am Tage als auch während der Nacht signifikant verringert. Die Eltern der Budenosid-behandelten Kinder gaben zu $89 \%$ an, das Asthma habe sich gebessert, in der Plazebo-Gruppe kamen $44 \%$ zu diesem Urteil $(p<0,005)$.

Auch nach der Follow-up-Phase nach weiteren 12 Wochen waren unter der inhalativen Kortikoidtherapie noch $28 \%$ der Kinder frei von Exazerbationen, in der Plazebo-Gruppe dagegen $0 \%(\mathrm{p}<0,05)$.

Nebenwirkungen traten in der $\mathrm{Bu}$ denosid-Gruppe bei 11, in der Plazebo-Gruppe bei 15 Kindern auf. Unter
Budenosid kam es in je einem Fall zu oraler Candidiasis und transienter Hyperexzitabilität.

Die Autoren kommen zu dem Schluß, daß die inhalative Behandlung mit vernebeltem Budenosid (1 mg zweimal täglich) bei Kindern mit schwerem Asthma effektiv und verträglich ist.

(zo)

\section{Kommentar}

Die Behandlung des frühkindlichen Asthma bronchiale ist schwierig, nicht zuletzt wegen des Problems, die Erkrankung in dieser Altersgruppe zu definieren. Giemende Atmung und Dyspnoe als Reaktion auf eine virale Infektion oder durch weitere auslösende Faktoren verursacht, reflektieren entzündliche Veränderungen der Atemwege.

Die vorliegende kontrollierte Studie zeigt, daß in der BudenosidGruppe im Vergleich zur PlazeboGruppe signifikant weniger Exazerbationen auftraten, die Dauer der oralen Steroidtherapie signifikant kürzer und die Inzidenz des Giemens während des Tages als auch nachts signifikant geringer war. In dieser Altersgruppe ist die Wirksamkeit von vernebeltem Budenosid damit gut belegt. Zusätzliche Studien könnten diese Therapieform weiter sichern und etablieren.

de Blic J, Delacourt C, Le Bourgeois M, Mahut B, Ostinelli J, Caswell C, Scheinmann P. Efficacy of nebulized budenoside in treatment of severe infantile asthma: a double-blind study. J Allergy Clin Immunol 1996; 98: 14-20.

\section{Korrespondenzadresse:}

Dr. med. R. Kehrt, Med. Fakultät der Humboldt-Universität zu Berlin, Virchow-Klinikum, Abt. Pädiatrie, Augustenburger Platz 1, D-13353 Berlin. 